\title{
CareLex eTMF Terminology
}

National Cancer Institute

\section{Source}

National Cancer Institute. CareLex eTMFTerminology. NCI Thesaurus. Code C114548.

A controlled vocabulary developed to support content interoperability between electronic

Trial Master File systems for clinical trials. 